\title{
A General Framework of LSTM and Transfer Learning Based CFDAMA Strategy in Broadband Satellite System
}

\author{
Qiang $\mathrm{He}^{*}$, Zheng Xiang and Peng Ren \\ School of Telecommunications Engineering, Xidian University, Xi'an 710071, \\ China \\ E-mail: bchqiangu@126.com \\ ${ }^{*}$ Corresponding Author
}

Received 20 November 2020; Accepted 11 December 2020;

Publication 09 March 2021

\begin{abstract}
The speeding development of wireless communication technology not only provides people with increasingly available bandwidth but also makes itself presents a trend of being heterogeneous, wide-band. Nowadays, because of its broadband service capability and wide coverage, satellite networks have been paid more and more attention. This paper investigates the framework of a long-short term memory (LSTM) and transfer learning-based Combined Free/Demand Assignment Multiple Access (CFDAMA) scheme (CFDAMALSTMTL), which is verified as an effective multiple access control scheme in the broadband satellite system. As we know, there is a delay time T between the user sending a request to the satellite and receiving a reply from the satellite, and the traditional multiple access control schemes has not processed the data traffic generated in this period of time. In this paper, we propose a new prediction strategy, which combines LSTM with transfer learning, to predict the data generated in this period. We introduce the prediction method into the CFDAMA scheme so that it can reduce data accumulation by the way of sending the sum slots requested by the user and the predicted request slots generated in this delay time. In the experimental verification, we give
\end{abstract}

Journal of Web Engineering, Vol. 20_2, 431-460.

doi: 10.13052/jwe1540-9589.2029

(C) 2021 River Publishers 
the performance comparison of CFDAMA-LSTMTL, CFDAMA-PA, and CFDAMA-PB, which gives the effectiveness of the CFDAMA-LSTMTL in a broadband satellite system.

Keywords: Multiple access control scheme, long-short term memory, transfer learning, prediction based strategy.

\section{Introduction}

With the rapid development of modern wireless communication technology, broadband is in great demand. As we all know, terrestrial mobile communication networks have been able to meet the needs of wide bandwidth communication for users in many areas with a very low cost [1]. However, the terrestrial mobile communication system can not meet the communication requirements of remote areas. The main reason why the service could not be established for these areas is that we can not deploy terrestrial communication system conveniently and economically. In that respect, although the terrestrial mobile communication system has the advantage of wide coverage, satellite networks are regarded as an indispensable and complementary communication method in future communication systems [2-4].

In the last two decades, great improvements have been made in satellite communication technology. In research and industry, multiple access control scheme is one of the key technologies in the satellite communication networks. Because it can provide high performance of QoS at a very low cost, the satellite communication networks have been applied more and more widely. In the coming $5 \mathrm{G}$ or even $6 \mathrm{G}$ era, the satellite communication networks are regarded as one of the most important part of the new networks. In the future integrated network, satellite networks have been viewed as a core technology for smart cities, internet of things (IoT), wireless sensor networks (WSN), vehicular networking technology (VNT), and so on [5]. In this context, the next-generation satellite networks are expected to be an integrated networks which can help to provide global services, support innovative communication scenarios, and reduce operational costs et al. Practice has proved that satellite communication networks can not only improve the communication efficiency, but also meet the growing number of users within limited resources, especially along with the emerging techniques, such as MIMO (Multiple-input Multiple-Output), OFDM (orthogonal frequency division multiplexing), and cognitive radio (CR).

It is important to note that the satellite bandwidth is a limited and expensive commodity, and the widespread and long-term usage of the satellite 
Table 1 Typical multiple access model

\begin{tabular}{|c|c|c|c|}
\hline Category & Scheme & Key Characteristics & Drawback \\
\hline Fixed assignment & $\begin{array}{l}\text { FDMA } \\
\text { TDMA } \\
\text { CDMA }\end{array}$ & $\begin{array}{l}\text { Users send messages in } \\
\text { different } \\
\text { frequency/time/code } \\
\text { domains }\end{array}$ & $\begin{array}{l}\text { - Inefficient resource } \\
\text { utilization } \\
\text { - Low system } \\
\text { capacity }\end{array}$ \\
\hline Random assignment & ALOHA & $\begin{array}{l}\text { Users send data directly } \\
\text { when they need to }\end{array}$ & $\begin{array}{l}\text { - Data collision prone } \\
\text { - No guarantee in } \\
\text { QoS }\end{array}$ \\
\hline Demand assignment & DAMA & $\begin{array}{l}\text { Dynamic allocation of } \\
\text { resources according to } \\
\text { user's requests }\end{array}$ & $\begin{array}{l}\text { - Additional } \\
\text { allocation of } \\
\text { scheduled resources } \\
\text { is required } \\
\text { - Time slots need to } \\
\text { be captured for an } \\
\text { implicit reservation }\end{array}$ \\
\hline
\end{tabular}

communication networks will be limited by this factor. So, the multiple access control scheme is the focus of this study. So far, the main multiple access control protocols of satellite communication systems is OMA (orthogonal multiple access scheme), which are shown in Table 1 [6]. As shown in this table, the OMA can be divided into three categories. The frequency-division multiple access (FDMA), time-division multiple access (TDMA), code-division multiple access (CDMA), and space-division multiple access (SDMA) are fixed assignment protocols, while ALOHA and demand assignment multiple access (DAMA) schemes are random assignment and a demand assignment, respectively. The fixed assignment protocols, which are FDMA, TDMA, CDMA, and SDMA schemes, can provide access services when the number of users is small and can effectively avoid multiple access interference. For the OMAs, the orthogonality between users limits the number of users in the system and reduces the spectrum efficiency of the system. A great example of using of ALOHA protocol is that if we use the ALOHA protocol in a network with a high number of users, there will be a lot of conflicts in the network. Moreover, the use of DAMA requires network resource occupation. As the traditional multiple access protocols can not meet the needs of future network, there is an urgent need for new multiple access schemes, which can harmoniously effectively improve the system capacity. Therefore, this paper proposes a new multiple access protocols which can be harmoniously integrated with OMA, the motivation is to design a new multiple access control protocol for the existing satellite networks. 
Recently, the combined free demand assignment multiple access (CFDAMA) has emerged as an effective multiple access strategy. The CFDAMA scheme, which is proposed by Mohammed et al., is a TDMA based access scheme [7]. This new scheme is designed to provide significant improvements in the delay/utilization performance of satellite channels supporting a finite number of users with burst data traffic. The CFDAMA scheme, which is a combination of free assignment of time slots and demand assignment can help to provide a minimum end-to-end delay for a satellite communication network.

Due to the limitation of satellite bandwidth resources, the satellite communication networks will face much more severe challenges when the number of users and their data traffic is large [8]. The terrestrial networks and satellite networks have its advantages and disadvantages. The terrestrial networks can provide high transmission bandwidth at a low cost, while the satellite networks can provide the services for users that cannot be served by the terrestrial networks. It is very important for a communication network that it can provide a ubiquitous coverage. Therefore, satellite networks must provide convenient access capabilities no matter whenever and wherever. However, the existing satellite network is difficult to meet the needs of users, so there is still a lot of room to improve the performance of the existing multiple access algorithm.

In order to solve the existing problems mentioned above, this paper proposes a LSTM and transfer learning based CFDAMA scheme which is named CFDAMA-LSTMTL in the broadband satellite system. The main idea of this scheme is to improve the performance of the multiple access scheme in the broadband satellite system by the advantages of LSTM and transfer learning, which can be used to predict the data traffic in the network. The contributions of this paper are summarized as follows:

(1) In order to send the data generated by the network in time, a new strategy, which is used to solve the problem of data accumulation in the period of time $\mathrm{T}$, is introduced. The traditional multiple access schemes have not processed the data traffic generated in this period of time, which will increase the data accumulation in the satellite on-board queue. So, in order to transmit the data traffic in time, we propose a new strategy, which can help to predict the data generate in this processing time.

(2) We propose a new prediction strategy which combines LSTM with transfer learning. This new strategy can improve the performance of the prediction for the LSTM network when the period time of the data traffic 
increases (the prediction efficiency of the LSTM network becomes worse when the period time of the data traffic increases). Meanwhile, the characteristics of the prediction network can be transferred by the transfer learning scheme.

The rest of this paper is organized as follows. In Section 2, some preliminary studies will be present, firstly, and then we will introduce the related works. In Section 3, we will introduce some basic concepts of LSTM and transfer learning, and then propose the CFDAMA-LSTMTL scheme in detail. We will present the experimental results and analyze the results in Section 4. Section 5 will draw a summary of this paper and outline the future research directions.

\section{Preliminary Studies and Related Works}

The multiple access control (MAC) protocols is very important for the satellite communication system, and CFDAMA is one of them which has been widely used in broadband satellite networks. The basic principle of the CFDAMA scheme can be descript as: it assigns the slots in a frame to the user on-demand, firstly; secondly, it assigns the remaining slots to the user in the system one by one in a freeway. With these two steps, it can ensure the rationality and fairness for resource assignment of the satellite. Due to the advantages of on-demand assignment and free assignment, the CFDAMA scheme can provide a better comprehensive performance in the broadband satellite networks.

\subsection{Basic Principle of CFDAMA}

A scheduler, which is always been deployed on the satellite or a hub station on the ground, is important for a satellite communication system. In this network, it is assumed that there is a satellite onboard scheduler, by which the user can access the satellite communication system in a short time. As mentioned above, the basic principle of CFDAMA strategy is to combine demand assignment and free assignment. With the help of the CFDAMA scheme, it can provide a low delay in low channel load and a high channel utilization in high channel load. The CFDAMA scheduler maintains a reservation request table and a free assignment table. The reservation request table, which is shown in Table 2, queues requests for users according to the demands. According to this table, it will place an entry on the bottom of the reservation request table which indicating the identity (ID) of the requesting user and the 
Table 2 Reservation request table

\begin{tabular}{lll}
\hline ID of user 0 & Number of slots requested by user 0 & Number of slots assigned to user 0 \\
ID of user 1 & Number of slots requested by user 1 & Number of slots assigned to user 1 \\
ID of user 2 & Number of slots requested by user 2 & Number of slots assigned to user 2 \\
\cline { 3 - 4 } & & \\
\cline { 3 - 4 } ID of user N & Number of slots requested by user N & Number of slots assigned to user N \\
\hline
\end{tabular}

Table 3 Free assignment table

\begin{tabular}{ll}
\hline ID of user 0 & Number of free slots assigned to user 0 \\
ID of user 1 & Number of free slots assigned to user 1 \\
ID of user 2 & Number of free slots assigned to user 2 \\
\hline ID of user M & Number of free slots assigned to user N \\
\hline
\end{tabular}

corresponding number of slots requested once a request is received by the scheduler. The free assignment table, which is shown in Table 3, consists of the ID numbers of all active terminals in the system.

The satellite on-board scheduler allocates slots frame-by-frame, and it will transmit the information on the Time Division Multiplex (TDM) downlink. First of all, the scheduler will serve the entries from the top of the reservation request table by requesting the continuous slots to the corresponding users based on the number of slots requested. In the absence of any queued requests, the scheduler will freely assign slots to users in a circular manner. This is achieved by assigning successive slots, one by one, to the user currently at the head of the free assignment table, moving each user to the bottom of the table after each slot allocation. In order to provide users who have not received slots for a long time with a better chance to get free assigned slot, every time users receive on-demand assigned slots and delete them from the reservation request table, they will also move them to the bottom of the free assignment table.

Figure 1 shows the uplink/downlink frame format of the CFDAMA scheme. In this figure, RD is the reservation data, and UD is the user data [9]. The uplink frame includes the reservation data and the user data. The reservation data is used to load the reservation requests issued by the user, and the user data is used to load the data sent by the user. The satellite on-board scheduler allocates the number of slots to users on demand or freely, and then users send data in the corresponding slots allocated to them. 


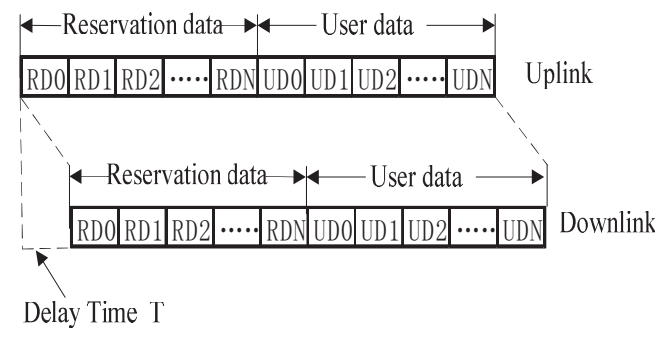

Figure 1 Uplink/downlink frame format of CFDAMA.

The downlink frame also includes the reservation data and user data, in which the reservation data is used to load the response information allocated by the satellite onboard scheduler to all the reservation requests. This information includes the user's data transmission slots and the free assignment information assigned by the satellite. User data is also used for loading the data sent by users. There is a time delay between the uplink and downlink frames, which represents the satellite on-board processing time.

\subsection{Related Works}

There are many improved CFDAMA schemes proposed in these years [10]. This section introduces four typical modified CFDAMA strategies, which are CFDAMA-PA (Pre-Assigned Request), CFDAMA-RA (Random Access Request), CFDAMA-PB (Piggy-Backing Request), and CFDAMA-RR (Round Robin Request). A more detailed description of the mentioned four strategies is described as follows.

\subsubsection{CFDAMA-PA}

The CFDAMA-PA, which is the abbreviation of CFDAMA-Pre Assigned scheme, is the CFDAMA scheme based on scheduled slots [11]. The uplink/downlink frame format of CFDAMA-PA is just the same as the CFDAMA scheme. In a satellite network with the CFDAMA-PA scheme, all users have their own fixed request slots in the uplink frame, and all users send their requests in its fixed slots. When there is slot request, each user will make a request. The number of slots requested by the user is given by formula (1).

$$
\mathrm{NSR}=\mathrm{NPQ}-\mathrm{NOR}
$$

$\mathrm{NSR}=$ Number of slots requested

$\mathrm{NPQ}=$ Number of packets queued

$\mathrm{NOR}=$ Number of outstanding requests 


\subsubsection{CFDAMA-RA}

The CFDAMA-RA, which is the abbreviation of CFDAMA-Random Access, is the CFDAMA scheme based on random access request slots [12]. In a satellite network with the CFDAMA-RA scheme, the uplink frame incorporates a region of random access request slots at the start of each frame, in which the users can send the requests by ALOHA within the slots by competitive request. The downlink frame, which is sent by a satellite, contains the reply data of the slots requested by users, the reply data of the data transmission slots, and the downlink traffic data. Due to the random competition in the uplink request process, different users may conflict when sending requests at the same time. When there is a conflict, the user needs to retransmit the data. Therefore, there is an ACK packets for the downlink data that sent by the satellite which is used to inform the success of the request.

All the users in this system are arranged to send requests to satellites in a random access request slot. According to this scheme, the number of slots requested by the user can be denoted by formula (2).

$$
\mathrm{NSR}=\mathrm{NPQ}-\mathrm{NOR}
$$

\subsubsection{CFDAMA-PB}

The CFDAMA-PB, which is the abbreviation of CFDAMA-Piggy Backing, is the CFDAMA scheme based on piggyback reservation [13]. The uplink frame format of this strategy is atypical. In the CFDAMA-PB scheme, request slots are embedded with the uplink data transmission slots. The users make requests accompanying their data packets transmission. If required, the user will make a request accompanying its data packets transmission. The number of slots required by users is given by formula (3).

$$
\mathrm{NSR}=(\mathrm{NPQ}-1)-\mathrm{NOR}
$$

\subsubsection{CFDAMA-RR}

The CFDAMA-RR, which the abbreviation of CFDAMA-Round Robin, is the CFDAMA scheme based on polling reservations [14]. In the CFDAMARR scheme, all users send requests by polling. The polling order is determined the data sent by satellite in the downlink frame.

According to the CFDAMA-RR scheme, there is not only a reservation request table and a free assignment table, but there is also a polling table, which is used to assign the order of polling assignment in the satellite scheduler. The contents of the polling table is the same as the free assignment table, which contains the ID and the number of slots assigned to the corresponding 
user in the system. The uplink frame consists of a series of polling assignment slots and the uplink data traffic slots. The satellite onboard scheduler assigns the uplink traffic slots on-demand/freely.

The user sends requests to the satellite in the polling assignment slots assigned by the satellite, and the number of the polling assignment slots of the user is obtained by formula (4).

$$
\mathrm{NSR}=\mathrm{NPQ}-\mathrm{NOR}
$$

\subsection{Focal Point}

According to the traditional CFDAMA scheme, the satellite on-board scheduler fills the requests into the resource assignment table (reservation request table and free assignment table) according to the first-in-first-out principle, and then the scheduler will provide service from the top of the reservation request table by demand assigning contiguous slots to the corresponding user, based on the number of slots requested. In the free assignment phase, it is achieved by assigning successive time slots one by one to the users at the head of the free allocation table. After each time slot is allocated, each user is moved to the bottom of the table. As a result, you can imagine that with the increasing numbers of users and data traffic, not all users can get the sending resources in time. Besides, the user who need more slots may be placed at the back of the resource assignment table, thus the slots assignment can not be obtained, which will also cause the accumulation of data traffic and the increase of mean end-to-end delay. In practical applications, the types of services requested by users mainly include real-time services and non-real-time services, in which real-time services mainly include voice and non-real-time services mainly include data services. Different type of service has different requirements of network quality of service (QoS). According to the queuing strategy used in this system, the priority of real-time services is higher than that of non-real-time services, and the priority of service with a large amount of data is higher than that of service with a small amount of data. The higher the priority of the user, the higher the user's position in the reservation request table. With the help of this new strategy, users with a large demand for real-time service and slots can obtain faster slots assignment and higher slots assignment performance.

As mentioned above, there is a time delay $\mathrm{T}$ between the uplink and downlink frames, which is the satellite onboard processing time. During this period, if the terminal is still in a continuous burst data process, the slot request from the users will be queued in the satellite onboard buffer which 
is also called the resources request queue. However, the existing multiple access protocols do not process the data generated in this period. So, with the increase of the number of users and the flow of users, data packets will be accumulated in the queue, which will decline the performance of the satellite networks. The main research is the prediction of the data generated in the satellite onboard processing time. By this prediction strategy, if the current user is in a continuous burst state, we can predict the amount of data packets that will arrive in the time delay. The total number of slots requested by the user is the sum of the current number of slots and the number of slots obtained by the prediction. With the help of this prediction strategy, the accumulation of data packets in the queue can be well addressed.

\section{LSTM and Transfer Learning based CFDAMA Scheme}

Machine learning, which is a science of getting computers to act without being explicitly programmed, has shown a better performance in data prediction. It can help to mine the hidden rules from a large amount of data and use it for data prediction [15]. However, several studies showed that a prediction model built by traditional machine learning leaves much room to be improved especially when the training samples and the predicted samples fail to meet the independent identical distribution hypothesis. Long ShortTerm Memory (LSTM) is a typical machine learning method which can solve the long-term dependence problem of general RNN. LSTM has a good performance in big data analysis, prediction, control, and some other fields. The practice has shown that transfer learning performs very well in learning fields, especially when the distribution of data used in training and testing are different from each other [16]. Nowadays, both theory and practice have proved that transfer learning is becoming a powerful weapon to deal with these learning problems. However, the prediction efficiency of the LSTM network becomes worse when the time span of the data traffic increases which can be improved by transfer learning. So, in this paper, we propose a new prediction strategy that combines LSTM with transfer learning to improve the prediction performance. Our motivation is to propose an effective data prediction method and use it to predict the data generated in the on-board processing time.

\subsection{Principle of LSTM}

As mentioned above, Long Short-Term Memory (LSTM), which is proposed in 1997 by Sepp Hochreiter et al., is a deep learning method [17]. LSTM is an 


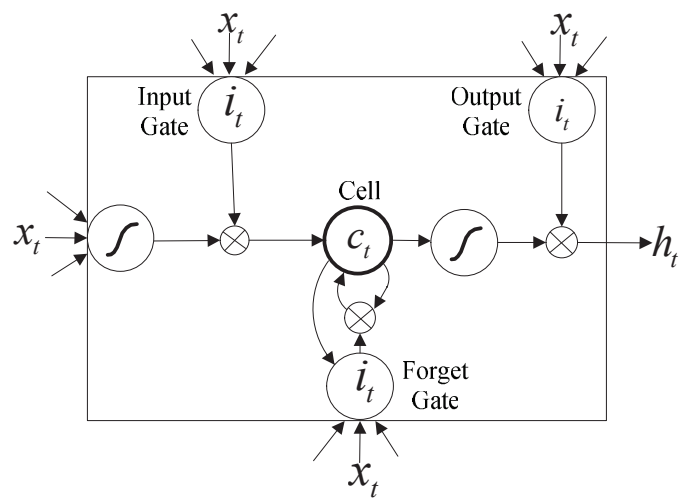

Figure 2 The basic structure of LSTM.

improved model of the traditional neural network model, which has a number of layers of nonlinear transformation. With the modified model, LSTM can create conditions for more complex task modeling. Machine learning, which is a potential data processing method, can help to dig out the potential information contained in the data when there are sufficient training samples.

In order to introduce LSTM into this system, we should construct a new deep neural network which has the ability of deep learning. Same as RNN, LSTM network always consists of three network layers, which contains an input layer, an output layer, and some of the hidden layers that accordance with different situations [18]. The basic structure of the LSTM network is shown in Figure 2. As shown in this figure, the hidden layer is composed of some memory cells. By this figure, we can see that one cell consists of three gates, which are input gate, forget gate and output gate, and a recurrent connection unit. In this network, the input is $x_{t}$, and $s_{t}$ is the state update function. Then, the current output is $o_{t}$, and $c_{t}$ is the internal memory of the unit. $g$ is a sigmoid activation function, while input and cell state are often transformed with the function of $\tanh ($.$) . So, LSTM cell can be defined as$ formula (5)-(10) [19].

Input gate:

$$
i_{t}=g\left(W_{x i} x_{t}+W_{h i} s_{t-1}+b_{i}\right)
$$

Forget gate:

$$
f_{t}=g\left(W_{x f} x_{t}+W_{h f} s_{t-1}+b_{f}\right)
$$

Output gate:

$$
o_{t}=g\left(W_{x o} x_{t}+W_{h o} s_{t-1}+b_{o}\right)
$$




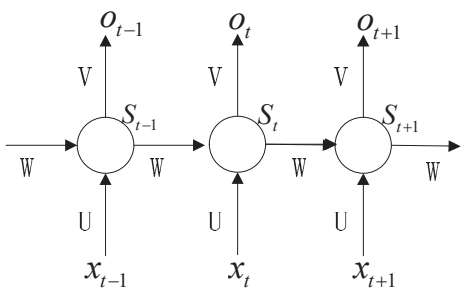

Figure 3 Network structure unfolded in time.

Input transform:

$$
c_{-} i n_{t}=\tanh \left(W_{x c} x_{t}+W_{h c} s_{t-1}+b_{c \_i n}\right)
$$

State update:

$$
\begin{aligned}
& c_{t}=f_{t} \cdot c_{t-1}+i_{t} \cdot c_{-} i n_{t} \\
& s_{t}=o_{t} \cdot \tanh \left(c_{t}\right)
\end{aligned}
$$

where $W_{i j}$ is the connection weights of neuron $i$ to $j, b$ is deflection.

It is very important to get the number of hidden layers and neurons in each layer. They are the key factors in the neural network training model, which can impact the prediction accuracy of this network. Theoretically, the more hidden layers, the more complex the network will be, but the prediction performance of the network will be better. Structurally, LSTM is a modified RNN (recurrent neural network) architecture, and the network structure of LSTM can be found in Figure 3. In this figure, $x_{0}, x_{1}, \ldots, x_{t+1}$ is the input vector, $o_{0}, o_{1}, \ldots, o_{t+1}$ is the output vector, $U, V, W$ are the network parameters.

In general, there are three important steps in the LSTM networks to establish the prediction network. Step 1 is to selectively forget the input of the previous node, step 2 is to selectively store the input parameters, and step 3 is to output the result.

\subsection{LSTM based Data Prediction Strategy}

Theoretical and practical studies have proved that the packet data of highspeed network traffic usually presents obvious self-similarity characteristics, so the time series prediction model can better predict the actual traffic in the network.

In order to establish a prediction model, the historical traffic data are always selected as samples. According to Figure 3, the predicted data can 


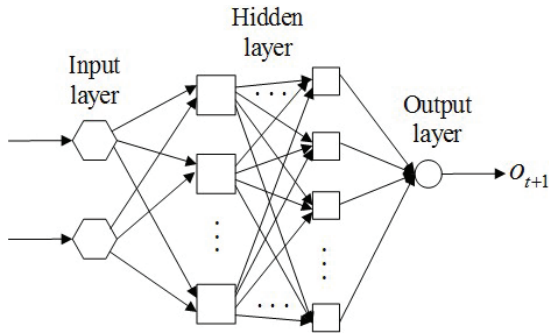

Figure 4 Prediction model and network structure of LSTM.

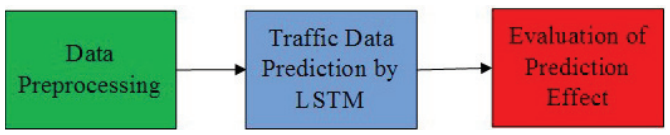

Figure 5 Data prediction based on LSTM.

be expressed as formula (11)

$$
o(t+1)=F(o(t), o(t-1), \ldots, o(t-n))
$$

where $o(t+1)$ is the predicted traffic data of a new time, o(t),o(t$1), \ldots, o(t-n)$ are historical data that contains the current and past traffic data. The prediction model and network structure of LSTM is shown in Figure 4. In this model, we can see that it has a basic structure like the RNN, and the predicted data traffic is the output. Then, Figure 5. shows the flow of data traffic prediction based on LSTM. There are three steps in this model, which are data preprocessing, traffic data prediction by LSTM, and evaluation of prediction effect. Then, we will give these three steps in detail.

\subsubsection{Data preprocessing}

The traditional data processing methods had encountered a bottleneck when dealing with a large number of data. Therefore, a new method is needed to improve the processing performance. In this system, the input data are usually standardized. The data standardization is to scale data to a small particular range, which means to convert the data into dimensionless pure values. After the data preprocessing, all these data that with different unit or scale of the indicator can be compared or weighted in the same domain.

In this paper, a Z-SCORE method, which can standardize the mean and standard deviation of the original data, is adopted [20]. After the data standardization, the dataset meets the standard normal distribution. That is to say, the mean value of these data is 0 , and the standard deviation of these 
data is 1 . The method of data standardization used in this paper, which can calculates the difference between the actual value and the average value, and then divides it by the standard deviation, can convert all the data into the regional value near zero. Nowadays, this standardization method has been widely used, including the cases that the data distribution is unknown, the data are discrete, and the maximum and minimum may shift to the normal range and so on. The standardization method can be defined as the follow formula:

$$
Y(x)=\frac{x-\bar{x}}{\sigma}
$$

where $\bar{x}$ is the average of all elements, $\sigma$ is the standard deviation of all elements.

\subsubsection{Data Prediction based on LSTM}

In recent years, data prediction scheme has been greatly developed. Generally speaking, Physical methods, statistical methods, learning methods, and combinations of these three methods are common methods for data traffic prediction. Different methods have their own characteristics and different performances. At present, the learning method is widely used in data prediction. A learning method, which is used based on historical data, can make full use of historical data information to achieve better prediction performance. Similarly, as a typical learning method, LSTM can achieve effective prediction. The process of data traffic prediction by LSTM is described as follows.

Input: $x_{t-n}, x_{t-n+1}, \ldots, x_{t-k}, x_{t-k+1}$, which is the historical data traffic vector.

(1) Get the number of layers of the LSTM network. Generally speaking, the more layers of the LSTM network, the stronger the learning ability, and the better the prediction effect will be.

(2) Get the abandonment rate of each network node. In order to prevent over-fitting, the default value of this paper is set to 0.5 .

(3) Get the iteration updating method of weight parameters.

(4) Get the epoch and batch size of model training.

(5) Train the LSTM network by the parameters determined by the above steps and the input data.

(6) Predict the data traffic of $x_{t-k+2}$. 


\subsubsection{Evaluation method}

Evaluation method is very important for data prediction performance evaluation. In this paper, there are three evaluation parameters, which are RMSE (Root Mean Error), MAPE (Mean Absolute Percentage Error), and MAE (Mean Absolute Error). These evaluation parameters are given as (13)-(15). In these formulas, $N$ is the number of samples, $y_{t}$ is the actual value of time $t$, and $\hat{y}_{t}$ is the predicted value.

$$
\begin{aligned}
M A P E & =\frac{1}{N} \sum_{t=1}^{N}\left|\frac{y_{t}-\hat{y}_{t}}{y_{t}}\right| \times 100 \% \\
M A E & =\frac{1}{N} \sum_{t=1}^{N}\left|y_{t}-\hat{y}_{t}\right| \\
R M S E & =\sqrt{\frac{1}{N} \sum_{t=1}^{N}\left(y_{t}-\hat{y}_{t}\right)^{2}}
\end{aligned}
$$

\subsubsection{Overview of the LSTM Algorithm}

The pseudo-code for data traffic prediction based on LSTM is given in algorithm 1.

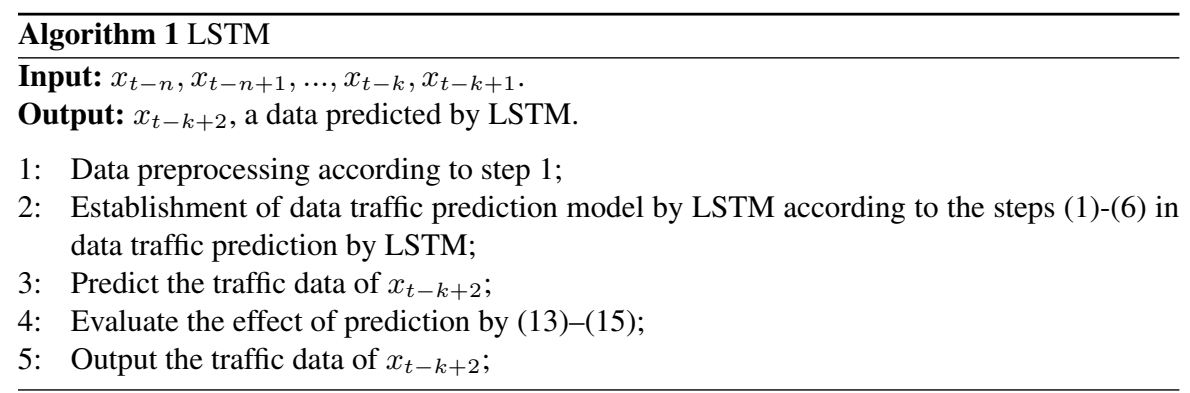

\subsection{Principle of Transfer Learning}

Traditional machine learning needs a lot of labeled data, which is a boring and expensive task, so transfer learning has attracted more and more attention. Transfer learning is a new machine learning method that uses existing 
knowledge to solve problems in different but related fields [21]. According to the transfer learning scheme, source domain and target domain data can have different distribution characteristics. Its aim is to transfer existing knowledge to solve the problem of learning with or without labels in the target domain. The basic principle of transfer learning is introduced as follows.

It is assumed that $D=\{x, P(x)\}$ is the source domain, and it can be divided into two parts:

(1) Feature space $x$;

(2) Marginal distribution probability $P(x)$;

Then, there is a learning task which is represented by $T=\{y, f\}$, and it can be divided into two parts too:

(3) Label space $y$;

(4) An objective prediction function $f$, which establishes the prediction of $\{x, y\}$.

For a specific learning process, we define the following parameters: source domain $D_{S}$ and learning task $T_{S}$, target domain $D_{t}$ and learning task $T_{t}$. The goal of transfer learning is to use the knowledge in $D_{S}$ and $T_{S}$, to improve the learning of target prediction function in $D_{t}$. $D_{S} \neq D_{t}$, or $T_{S} \neq T_{t}$.

When there are a lot of calibration data in the source domain, but only a few calibration data in the target domain, and the source domain and the target domain have some common generalization characteristics, the transfer learning can be used to apply the data pre-training model of the source domain to the task of the target domain to improve the performance of the model in the target domain. For the prediction of time series data, this paper uses the observation data of different time, combines transfer learning with LSTM, to predict the future data.

This paper proposes a new time series data transfer learning method which can make full use of the information of "historical data". According to this new scheme, the time series data transfer learning refers to train the LSTM network by the time series data, and then transfer the characteristic of the trained LSTM network to a new LSTM network and try to fine-tune the parameters of the new network by a new time series data. Finally, the prediction of future data can be get by this trained network. The principle of transfer learning in this paper is shown in Figure 6. 


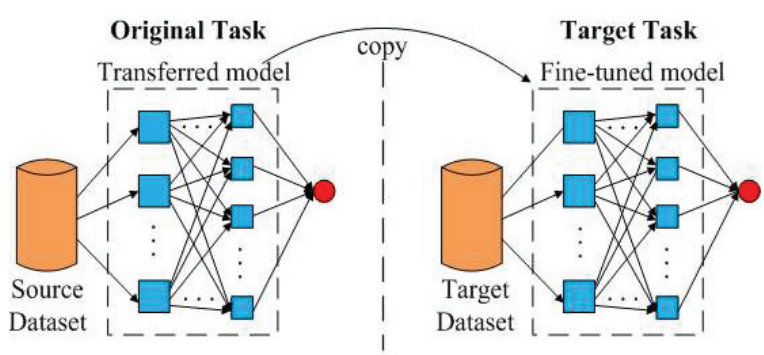

Figure 6 Principle of transfer learning.

\subsection{Resource Scheduling Strategy of the Satellite}

As mentioned above, scheduling algorithm is very important for data transmission. In this paper, we propose a new resource scheduling strategy, which can achieve priority ranking. Firstly, the satellite scheduler prioritizes the users according to the number of slots request, secondly, it sets the priority number for the users in this system. The higher the priority of the user who requests more slots, the higher the position in the on-demand allocation table, which ensures that the user who needs more slots can obtain faster slots allocation and higher slots assignment rights. With the help of this queuing idea, the mean end-to-end delay performance will be improved, especially for the high data traffic network.

\subsection{LSTM and Transfer Learning based CFDAMA Strategy}

In this paper, a new CFDAMA strategy, which is based on LSTM and transfer learning, is proposed, and it is abbreviated as CFDAMA-LSTMTL. The purpose of this strategy is to predict the traffic data that may be generated in the on-board processing time described above, so as to reduce the accumulation of data packets in the satellite network. The main steps of this strategy can be described as follows: firstly, the input data should be divided into several data sequences with a certain length of $l(l \leq n)$, and then sort them in time order. Secondly, a LSTM network should be established and trained by the first data sequence. Thirdly, the characteristic parameters of the trained LSTM network should be transfered to a new network and try to fine-tune the characteristic parameters of the new network by a new data sequence. Fourthly, recycle and fine-tune the new network until all data sequences are trained. Finally, if all these data sequences have been used to train the network, then we can predict the data traffic by the trained network. 
The pseudo-code of the CFDAMA-LSTMTL scheme is given in Algorithm 2 .

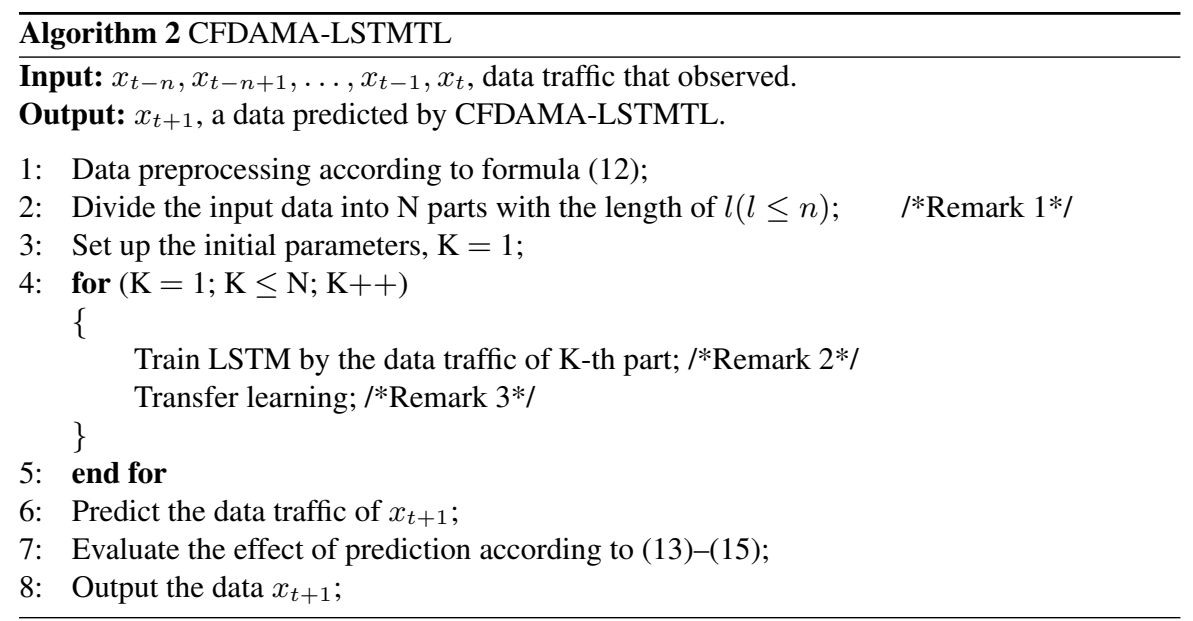

Remark 1: The first part of the input data is $x_{t-n}, x_{t-n+1}, \ldots, x_{t-n+l-2}$, $x_{t-n+l-1}$, the second part is $x_{t-n+l}, x_{t-n+l+1}, \ldots, x_{t-n+2 l-2}, x_{t-n+2 l-1}$, and then the N-th part is $x_{t-n+(N-1) l}, x_{t-n+(N-1) l+1}, \ldots, x_{t-1}, x_{t}$.

Remark 2: If $\mathrm{K}=1$, the first part of the input data is $x_{t-n}, x_{t-n+1}, \ldots$, $x_{t-n-l-2}, x_{t-n-l-1}$, and then we can train the LSTM network by these data.

Remark 3: It is one of the most important steps. In this step, we will transfer the characteristic parameters of the trained LSTM network to a new LSTM network and try to fine-tune the parameters of the new network by $(\mathrm{K}+1)$-th data traffic.

Figure 7 shows the principle of CFDAMA-LSTMTL scheme.

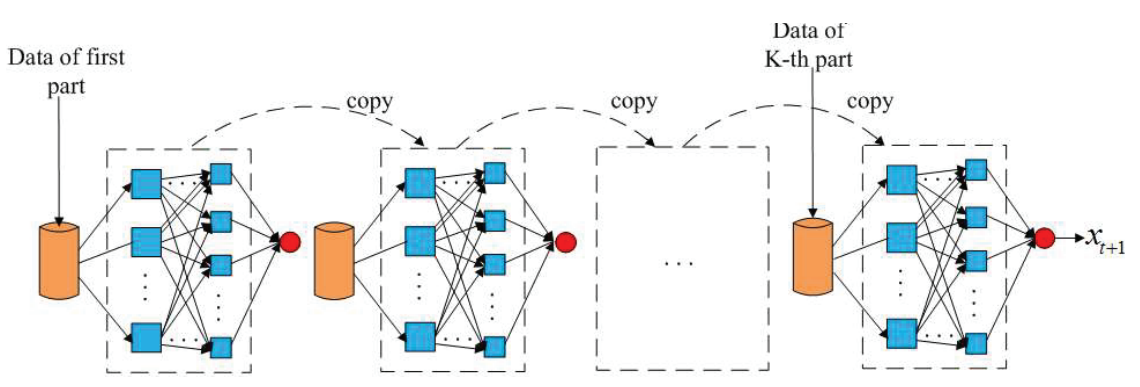

Figure 7 Principle of CFDAMA-LSTMTL. 
As mentioned above, the CFDAMA-LSTMTL is a CFDAMA scheme based on the combination of LSTM and transfer learning, and it can take the advantages of transfer learning and LSTM, which can not only use the "experience" but also can deal with the long sequences efficiently.

\section{Experiment and analysis}

In this section, there are many representative simulation results which can be used to verify the effectiveness of the proposed CFDAMA-LSTMTL scheme. The simulation results of this scheme are given as well as compared to the other two typical schemes CFDAMA-PA and CFDAMA-PB. In this section, these three schemes have been simulated by the combination of MATLAB, STK, and OPNET. Here, we use MATLAB to generate traffic data, and use STK to generate the satellite orbital data, and then we import the orbital data and traffic data into OPNET which can realize the network simulation.

\subsection{Data Traffic Model}

A large number of theories and practice have proved that a correct traffic model is important to evaluate the performance of the MAC protocol. As any differences between the models and actual traffic will lead to an overestimation or underestimation of the achievable performance, we should pay attention to the establishment of the right traffic model. In this paper, there is a Pareto ON-OFF traffic model in this simulation. Figure 8 gives the state transition diagram of the pareto ON-OFF model [22]. In this figure, we can see that the traffic model consists of several independents ON and OFF traffic durations, and the process of data packet generation is constantly changing between the state of ON and OFF. According to this model, the traffic data is generated when it is $\mathrm{ON}$, and the transfer probability, which means from ON to OFF, is $\beta_{1}$. There is no data when it is OFF, and the transfer probability, which means from OFF to $\mathrm{ON}$, is $\beta_{2}$. For the $\mathrm{ON}$ state, there is a distribution function which is used to determine the number of data packets, and the interval of the arrival of data packets also obeys a certain distribution.

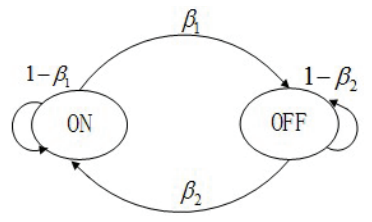

Figure 8 ON-OFF traffic model. 


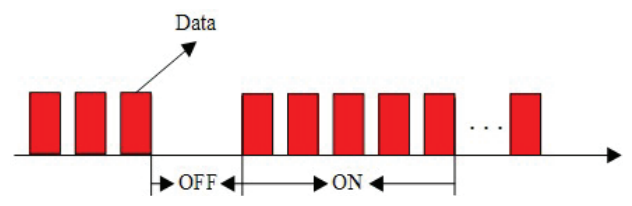

Figure 9 Pareto ON-OFF traffic data model.

It is demonstrated that the traffic data in modern high-speed networks has obvious self-similarity, which means that the data is bursty over a wide range of time scale tails. The state duration, which includes the interval of the arrival of data packets and the duration of packets, can not be described by the exponential distribution, while the Pareto distribution can well represent the arrival status of data packets. Therefore, the Pareto ON-OFF data traffic model is used in this experiment. The Pareto distribution can be described as formula (16). In this formula, $f_{O N}\left(t_{O N}\right)$ and $f_{O F F}\left(t_{O F F}\right)$ are the probability density function of ON and OFF state. $\lambda_{1}, \lambda_{2}, \varepsilon_{1}, \varepsilon_{2}$ are the parameters of time scale tails and minimum duration of $\mathrm{ON}$ and $\mathrm{OFF}$, respectively. $t_{O N}$ and $t_{O F F}$ are the parameters of state duration of ON and OFF. The data traffic model is shown in Figure 9.

$$
\begin{gathered}
f_{O N}\left(t_{O N}\right)=\lambda_{1} \varepsilon_{1}^{\lambda_{1}} t_{O N}^{-\lambda_{1}-1}, \lambda_{1}, \varepsilon_{1} \geq 0, t_{O N} \geq \varepsilon_{1} \\
f_{O F F}\left(t_{O F F}\right)=\lambda_{2} \varepsilon_{2}^{\lambda_{2}} t_{O F F}^{-\lambda_{2}-1}, \lambda_{2}, \varepsilon_{2} \geq 0, t_{O F F} \geq \varepsilon_{2}
\end{gathered}
$$

\subsection{Simulation Scenario}

In the simulation model, there are many Inmarsat synchronous satellites and ground nodes. Here, the satellite is used to provide ubiquitous communications services for users. The simplified system model of the simulation scenario is shown in Figure 10. According to the satellite networks shown in this figure, there is an onboard scheduler on the Inmarsat satellite, which can process and forward frames. The ground nodes consist of several users, which can communicate with each other with the help of the satellite and the multiple access protocol. In order to ensure the performance of communication, the number of satellite users can not be too large. The parameters for the broadband satellite system, which are used to build a virtual simulating environment, are given in Table 4. Then, the parameters for the LSTM network, which are used to build a LSTM network, are given in Table 5. 


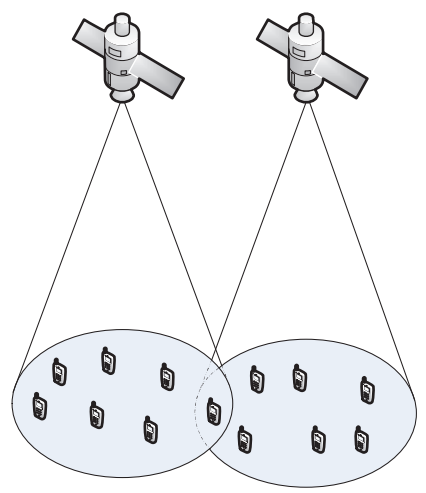

Figure 10 Simulation scenario.

Table 4 Simulation parameters for the satellite network works

\begin{tabular}{lc}
\hline Parameters & Setting \\
\hline Number of satellite & 12 \\
Altitude of satellite (km) & 35700 \\
Number of ground stations & $10,20,40,60,80$ \\
Channel rate (Mbit/s) & 2.2 \\
Number of Carriers per Frame & 8 \\
Number of slots per carrier & 128 \\
Number of bits per slot (bit) & 420 \\
Time length per frame (s) & 0.0027136 \\
Channel model & Shadowed-Rician fading \\
Channel Load & $0.1-1.0$ \\
Source Model & Poisson \\
& Pareto ON-OFF \\
Minimum duration of ON and OF & $\varepsilon_{1}=\varepsilon_{2}=1.0$ \\
Parameters of time scale tails & $\lambda_{1}=\lambda_{2}=1.2$ \\
\hline
\end{tabular}

\subsection{Experimental Results and Analysis}

In this paper, the performance of CFDAMA-LSTMTL scheme is compared with that of CFDAMA-PA and CFDAMA-PB schemes, and the experimental results and analysis are given. It is necessary to be emphasized is that the network parameters are the same in all our experiments. That is to say, for fair comparison, we have used the same parameters and we do not tune the parameters in all these experiments for these three different strategies. 
Table 5 Simulation parameters for the LSTM network

\begin{tabular}{lc}
\hline Parameters & Setting \\
\hline Number of input nodes & 8 \\
Number of output nodes & 24 \\
Time step & 72 \\
Number of Hidden Nodes & 1024 \\
Learning rate & initial value 7e-6 \\
Activation function & ReLU \\
Batch size & 24 \\
Number of iterations & 1024 \\
\hline
\end{tabular}

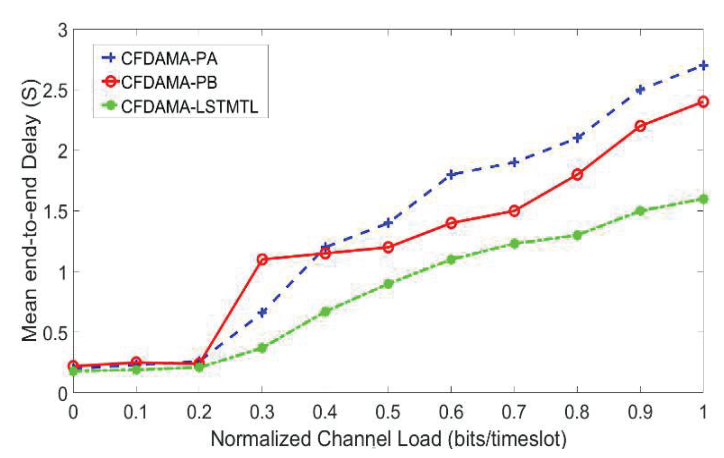

Figure 11 Comparison of mean end-to-end delay/normalized channel load.

Firstly, the comparison of mean end-to-end delay/normalized channel load performance, which can evaluate the mean end-to-end delay performance of these three schemes, is given. This experiment is with a fixed number of users but varying normalized channel load. Figure 11 shows the results of the mean end-to-end delay/normalized channel load, where the number of users is 20 and the channel load is between 0.1 and 1.0.

As can be seen from Figure 11, when the normalized channel load is between 0.1 and 0.2, the mean end-to-end delay/normalized channel load performance of these three schemes is essentially the same. After that, the performance of these three schemes increases sharply when the channel load is 0.2-1.0, and CFDAMA-PA and CFDAMA-PB increases much greater than CFDAMA-LSTMTL. The reason is that with the increases of normalized channel load, the burst data traffic increases, and the number of user's requests in the system will increase too. If the number of slots assigned on-demand at this time exceeds the total available slots of a frame, the users 


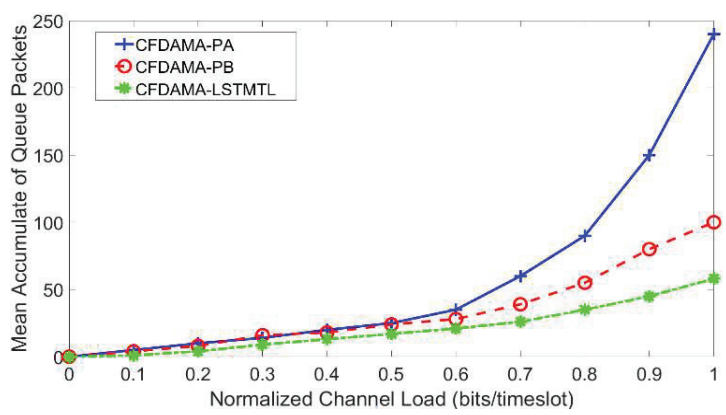

Figure 12 Comparison of mean accumulate of queue packets/normalized channel load.

with a large demand for slots behind the on-demand assignment table will not get enough slots, which will increase the mean end-to-end delay. The CFDAMA-PB scheme can increase the flexibility of slots assignment by piggybacking requests, which can avoid setting fixed slots to store user's requests at high normalized channel loads. However, if there are too many users, it will also lead to users with large slot requirements can not be assigned enough slots. In general, although the performance of CFDAMAPA is not as good as that of CFDAMA-LSTMTL, it is better than that of CFDAMA-PB. The CFDAMA-LSTMTL scheme assigns slots to users with slot requests when normalized channel load increases, and guarantees priority for data packets of users with large slots demand. Therefore, the mean end-to-end delay/normalized channel load performance is better than that of CFDAMA-PA and CFDAMA-PB.

Then, Figure 12 shows the comparison of the mean accumulate of queue packets/normalized channel load performance. According to this figure, the mean accumulate of queue packets for these three schemes is not much different in the range of 0.1-0.6, The reason might be that users can send the data in the queue in time when the channel load is small and the availability of system resources is high. From the normalized channel load of 0.6, the performance of these three schemes is different from each other. The performance of CFDAMA-PB and CFDAMA-LSTMTL is obviously superior to CFDAMA-PA, and CFDAMA-LSTMTL presents the best performance, which is because CFDAMA-PA has fixed slot points in the frame. In this system, it is possible that there are some users do not has requested when the next frame arrives, while a larger part of users have requests, but they can not get the corresponding slots to send. As for the CFDAMA-PB scheme, it does not take certain measures to the burst data traffic, and it can only 
deal with the data in the current queue in the case of high burst, which will result in the continuous accumulation of packets. In contrast, the CFDAMALSTMTL introduces the prediction strategy, it can predict the data traffic in the on board processing time, and if there is a burst of data, the number of slot requests send by a user at the current time is the sum of the number of slots for the existing burst data and the number of slots for the predicted data. For the CFDAMA-LSTMTL scheme, although it has a better performance, but the data accumulation will still increase when the channel load is high or reaches a certain threshold, which is because the number of current data packets exceeds the capacity of the whole system.

The experiment with the conditions of fixed channel load but a variable number of users, which can get the performance of these three schemes in access fairness, are given below. As shown in Figures 13 and 14, the channel load is 0.6 , and the number of users is $10,20,40,60$, and 80 respectively.

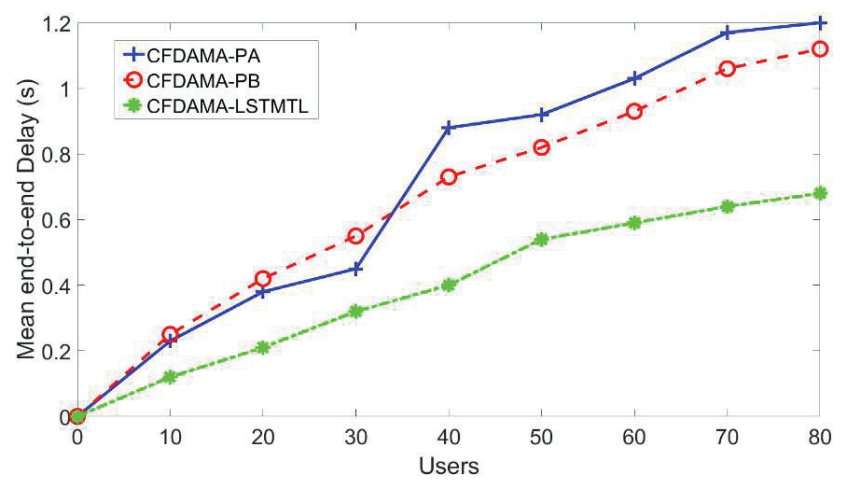

Figure 13 Comparison of mean end-to-end delay/number of users.

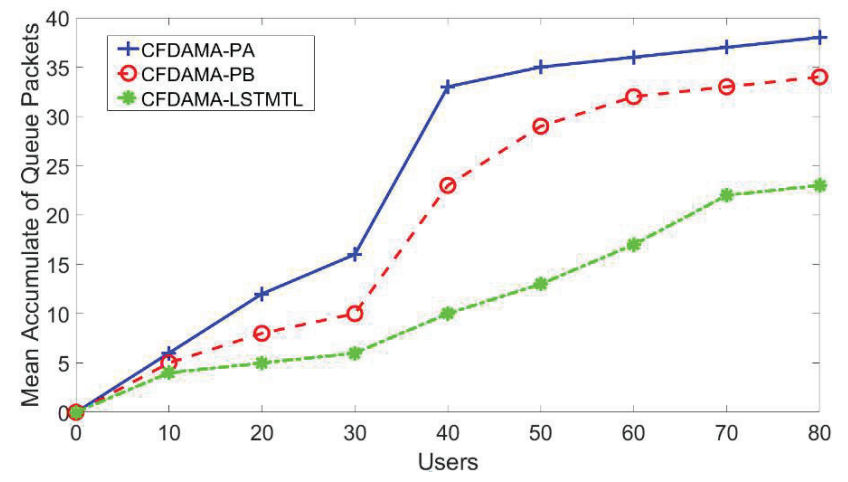

Figure 14 Comparison of mean accumulate of queue packets/number of users. 
The comparison of mean the end-to-end delay/number of users performance is given in Figure 13. It can be seen from it that the performance of CFDAMA-PA is similar to CFDAMA-PB in the range of 10-20 users. The reason is that when the number of users is small, CFDAMA-PA can provide timely access to the users by fixed appointment slots. The scheduling process of CFDAMA-PB is similar to CFDAMA-PA, and all of these schemes' fairness of users is also maximized. The mean end-to-end delay of the CFDAMA-LSTMTL scheme is slightly larger than that of the previous two schemes because of the data prediction process and computational sequencing.

At last, Figure 14 shows the results of the mean accumulate of queue packets/number of users. In this figure, we can see that the CFDAMALSTMTL scheme can guarantee the real-time and fairness of users' access by means of prediction of the data generated in the on board processing time. For the CFDAMA-PB scheme, it can guarantee the real-time and fairness of users' access by piggyback with the increasing number of users, which reduce the probability that users can not access in time in sudden situations and also reduce the data accumulation in the queues. Therefore, we can conclude that the CFDAMA-PB and CFDAMA-LSTMTL scheme can guarantee a better performance of real-time and fairness of users' access.

The above experiments prove the superior performance of the CFDAMALSTMTL scheme proposed in this paper. Meanwhile, in order to further evaluate the performance of the prediction strategy, we should pay attention to the evaluation method mentioned by formulas (13)-(15). Table 6 shows the evaluation results of the prediction performance of the proposed strategy. It can be seen from this table that the prediction performance of the proposed strategy for the training set and test set have shown improvements, which show a small deviation from the true value.

Both theoretical analysis and experimental research have proved that the proposed LSTM and transfer learning-based CFDAMA strategy, abbreviated as CFDAMA-LSTMTL, shows good performance. Compared with traditional multiple access protocol, this scheme has a better performance in end-to-end delay, average accumulate of queue packets and so on. So, we

Table 6 Evaluation results of the prediction performance of the proposed strategy

\begin{tabular}{lccc}
\hline Entry & RMSE & MAE & MAPE (\%) \\
\hline Training Set & 0.334 & 0.245 & 43.1 \\
Test Set & 0.265 & 0.134 & 27.4 \\
\hline
\end{tabular}


can conclude that the CFDAMA-LSTMTL scheme can gratify the multiple access requirements of the broadband satellite system.

\section{Conclusion}

This paper proposes a general framework of LSTM and transfer learning based CFDAMA strategy in broadband satellite system. The new strategy, which introduces the combination of LSTM and transfer learning, is abbreviated as CFDAMA-LSTMTL. The motivation of this new strategy is to predict the data traffic in the time of the satellite on-board processing, and then send the slot request which is the sum of the slot requested by a user and the slots predicted by the proposed scheme, so as to reduce the accumulation of data and promote the performance of multiple access in the broadband satellite system. Generally speaking, when a user needs to communicate with each other by the satellite networks, it will send a slot request to the satellite. However, there will be a delay time $\mathrm{T}$ which is also called onboard processing time. For the conventional multiple access schemes, the slots requested by the user do not include the data generated within that time, and it will cause the network performance to drop. In this paper, we propose a prediction strategy based on the combination of LSTM and transfer learning, which can predict the data in the on-board processing time. And then, we introduce the prediction strategy into the CFDAMA scheme and do some experiments to verify the performance of this strategy. The experimental results show that the CFDAMA-LSTMTL scheme performs better than CFDAMA-PA and CFDAMA-PB in both mean accumulate of queue packets/normalized channel load and mean end-to-end delay/number of users with the same network conditions.

\section{References}

[1] Gao, L., L. Chen, and F. Yu. "The Research on Effectiveness Evaluation of Mobile Broadband Wireless Communication System." Journal of Physics: Conference Series 1634.1,012061 (7pp), 2020.

[2] Liu, M., Z. G. Li, and Z. Dou. "Rainfall Attenuation Characteristic Analysis in Ka-band Satellite Communication System.” International Conference in Communications, Signal Processing, and Systems Springer, Singapore, pp. 1278-1285, 2017. 
[3] J. Zhang, et al., "Energy efficient hybrid satellite terrestrial 5G networks with software defined features," J. Commun. Netw., vol. 19, no. 2, pp. 147-161, 2017.

[4] Konstantinos, L., et al. "Use cases and scenarios of 5G integrated satellite-terrestrial networks for enhanced mobile broadband: The SaT5G approach." International Journal of Satellite Communications and Networking, pp. 1-22, 2017.

[5] Wang, N., et al. "Satellite Support for Enhanced Mobile Broadband Content Delivery in 5G." pp. 1-6, 2018.

[6] Yates, J. "Personal broadband satellite systems and services." Personal Broadband Satellite, IEEE Xplore, 2002.

[7] Tozer, T. C. "Performance of the combined free/demand assignment multiple access protocol with combined request strategies via satellite." Journal of Guangxi University for Nationalities, 2006.

[8] Xiangming Zhu, Chunxiao Jiang et al., "Non-Orthogonal Multiple Access Based Integrated Terrestrial-Satellite Networks," IEEE Journal on Selected Areas in Communications, vol. 35, no. 10, October. 2017.

[9] Le-Ngoc, T., and S. V. Krishnamurthy. "Performance of combined free/demand assignment multiple-access schemes in satellite communications." International Journal of Satellite Communications \& Networking, vol. 14, no. 1, pp. 11-21, 2015.

[10] P. D. Mitchell, D. Grace, T. C. Tozer, "Performance of the combined free/demand assignment multiple access protocol with combined request strategies via satellite,' IEEE Personal, Indoor and Mobile Radio Communications Conference (PIMRC), October. 2001.

[11] Mitchell, and P. Daniel. "Effective medium access control for geostationary satellite systems." University of York, 2003.

[12] T. Le-Ngoc, S. V. Krishnamurthy, "Performance of combined free/demand assignment multiple-access schemes in satellite communications," International Journal of Satellite Communications, vol. 14, no. 1, pp. 11-21, 1996.

[13] Li W. L., Zhou X., Zhao L. N. "Delay Performance of the CFDAMA-PB Protocol in the Different Source," Radio Communications Technology, vol. 21, no. 3, pp. 20-22, 2012.

[14] Gorma, W. M., and P. D. Mitchell. "Performance of the combined free/demand assignment multiple access protocol via underwater networks." International Conference on Underwater Networks \& Systems, ACM, pp. 1-2, 2017. 
[15] Tan, C., et al. "A Survey on Deep Transfer Learning." International Conference on Artificial Neural Networks, arXiv, 2018.

[16] Sainath, T. N., et al. "Convolutional, Long Short-Term Memory, fully connected Deep Neural Networks." ICASSP 2015 - 2015 IEEE International Conference on Acoustics, Speech and Signal Processing (ICASSP) IEEE, pp. 4580-4584, 2015.

[17] Schmidhuber, and Jürgen. "Deep Learning in Neural Networks: An Overview." Neural Netw, vol. 61, pp. 85-117, 2015.

[18] Xue Yusheng, Yu Chen, Zhao Junhua, et al., "A Review on Short-term and Ultra-short-term Wind Power Prediction," Automation of Electric Power Systems, vol. 39, no. 6, pp. 141-151, Mar. 2015.

[19] Zhang, J., et al. "Short-term forecasting and uncertainty analysis of wind turbine power based on long short-term memory network and Gaussian mixture model.” Applied Energy, vol. 241, pp. 229-244, 2019.

[20] Wenqi Ma. "Financial Pre-Warning of Company Basdon Neural Networks and Z-Score Model," Modern Management, vol. 7, no. 3, pp. 9399, 2017.

[21] R. Ribani, and M. Marengoni. "A Survey of Transfer Learning for Convolutional Neural Networks." 32nd SIBGRAPI Conference on Graphics, Patterns and Images Tutorials (SIBGRAPI-T) IEEE, 2019.

[22] Fei Liu, Janne Riihijä, et al., "Analysis of Proportional Fair Scheduling Under Bursty On-Off Traffic," IEEE Communications Letters, vol. 21, no. 5, pp. 1175-1178, 2017.

\section{Biographies}

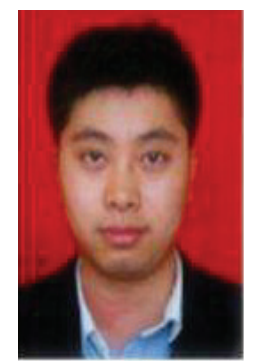

Qiang He was born in 1985. He received his B.S. degree in biomedical engineering from Sichuan University of Science \& Engineering, ZiGong, China, in 2010. He is currently pursuing the Ph.D. degree in science of military command, Xidian University, Xi'an, China. 


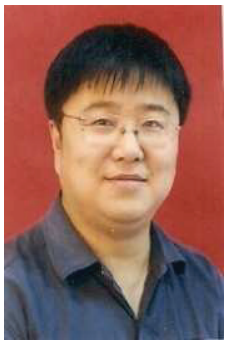

Zheng Xiang received the B.S. and M.S. degrees in Air Force Engineering University, Xi' an, China, in 1998 and the Ph.D. degree in Xi'an Jiaotong University, Xi' an, China, in 2006. Since 2002, he has been a Faculty Member with the School of Telecommunications Engineering, Xidian University, where he is currently a Full Professor with the State Key Laboratory of ISN. His current research interests include self-organizing networks, communication signal processing, and broadband data communication.

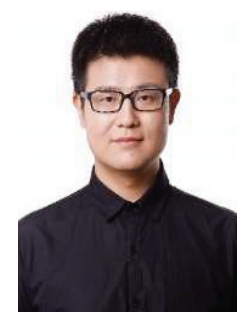

Peng Ren received the B.S., M.S., and Ph.D. degrees from Xidian University, Xi' an, China, in 2006, 2010, and 2014, respectively, all in telecommunications engineering. He is currently an Associate Professor with the School of Telecommunications Engineering, Xidian University, Xi' an, China. His current research interests are signal processing for wireless communications, Ad Hoc network, and multidimensional data analysis and processing. 
